OPEN ACCESS

Edited by:

Paul Takam Kamga, Université de Versailles SaintQuentin-en-Yvelines, France

Reviewed by: Konstantinos Linos, Dartmouth-Hitchcock Medical Center, United States

Giada Dal Collo,

Erasmus Medical Center, Netherlands

*Correspondence:

Yuchen Han

ychan@cmu.edu.cn

${ }^{\dagger}$ These authors have contributed equally to this work

Specialty section:

This article was submitted to

Thoracic Oncology,

a section of the journal

Frontiers in Oncology

Received: 08 April 2021 Accepted: 07 July 2021

Published: 20 July 2021

Citation:

Zhao J, Zhao R, Xiang C, Shao J, Guo $L$ and Han $Y(2021)$ YAP1 MAML2 Fusion as a Diagnostic Biomarker for Metaplastic Thymoma.

Front. Oncol. 11:692283.

doi: 10.3389/fonc.2021.692283

\section{YAP1-MAML2 Fusion as a Diagnostic Biomarker for Metaplastic Thymoma}

\author{
Jikai Zhao ${ }^{\dagger}$, Ruiying Zhao ${ }^{\dagger}$, Chan Xiang, Jinchen Shao, Lianying Guo and Yuchen Han * \\ Department of Pathology, Shanghai Chest Hospital, Shanghai Jiao Tong University, Shanghai, China
}

Background: Metaplastic thymoma is a very rare tumor with only a few case reports documented in literature. Hence, its molecular features have not been well explored.

Material and Methods: Seventeen specimens of metaplastic thymoma were sequenced and retrospectively analyzed by fluorescence in situ hybridization (FISH) and immunohistochemistry in the study. In addition, seven cases of micronodular thymoma with lymphoid stroma and nine cases of type A thymoma were also investigated.

Results: Among these metaplastic thymomas, fifteen cases showed classical histological features, and two cases displayed characteristic micronodular-like growth patterns. DNA and RNA based next-generation sequencing identified and confirmed highly recurrent Yes Associated Protein 1 (YAP1) - Mastermind Like Transcriptional Coactivator 2 (MAML2) translocation $(13 / 17,76.5 \%)$ in metaplastic thymoma but not in micronodular thymoma with lymphoid stroma $(0 / 7,0 \%)$ and type A thymoma (0/9, 0\%). In addition, six nonsense mutations were also detected in the metaplastic thymoma. FISH in microdissection specimens indicated that both epithelioid and spindle cell components harbored YAP1MAML2 gene rearrangements.

Conclusions: Our study explored the genetic alterations in epithelioid and spindle cell components in metaplastic thymoma. Furthermore, YAP1-MAML2 gene rearrangements emerged as a potential diagnostic biomarker helpful for distinguishing metaplastic thymoma from type A and micronodular thymoma with lymphoid stroma.

Keywords: metaplastic thymoma, YAP1-MAML2, sequencing, FISH, gene rearrangements, biomarker

\section{INTRODUCTION}

Metaplastic thymoma is an uncommon thymic epithelial malignancy that accounts for less than $1 \%$ of all types of thymomas (1). It is a relatively indolent neoplasm that shows biphasic differentiation and comprises of a solid growth epithelial cells along with gradual transiting spindle cells components (2). This terminology for the tumor was introduced in the World Health Organization (WHO) 2004 scheme and continued to the 2020 World Health Organization (WHO) classification for lung and thymus $(3,4)$.

Abbreviations: FISH, fluorescence in situ hybridization; YAP1, Yes Associated Protein 1; MAML2, Mastermind Like Transcriptional Coactivator 2; WHO, World Health Organization; NGS, next generation sequencing; H\&E, hematoxylin and eosin; MNTLS, micronodular thymoma with lymphoid stroma. 
To our knowledge, only rare cases of metaplastic thymoma have been reported in Asian population $(5,6)$, whereas about a total of 30 cases have been described separately in the English literatures $(7,8)$. The genomic alterations driving metaplastic thymoma have not previously been defined in any guidance or classification and hence little is known about its molecular characteristics. Recently, it had been reported that there are high frequency Yes Associated Protein 1 (YAP1) - Mastermind Like Transcriptional Coactivator 2 (MAML2) fusions in an eight metaplastic thymomas cohort study, and the genetic alterations may be closely related to tumor occurrence or prognosis (9).

In this study, we used a unified RNA and DNA nextgeneration sequencing (NGS) assay and Fluorescence in Situ Hybridization (FISH) to analyze the molecular characteristics of metaplastic thymoma and compared it with micronodular thymoma with lymphoid stroma as well as type A thymoma.

\section{MATERIAL AND METHODS}

\section{Patients and Specimens}

Seventeen cases of previously diagnosed metaplastic thymoma were collected by screening the archives of the Department of Pathology of Shanghai Chest Hospital from 2010 to 2019. The whole hematoxylin and eosin (H\&E) slides of all cases were reviewed by three pathologists with more than ten years of diagnostic experience on thoracic neoplasms. Since type A thymoma and micronodular thymoma with lymphoid stroma (MNTLS) may morphologically overlapped with metaplastic thymoma, we also selected 16 cases, including nine cases of type A thymoma and seven MNTLS. All the samples were surgically resected and their formalin-fixed and paraffinembedded (FFPE) specimens were used for the study. Clinical information, including age, sex, smoking status, tumor size, and Masaoka-Koga staging of all patients were collected from the medical records. Clinical follow-up data were collected from the consultations of clinicians or obtained from the medical records. The follow-up duration was calculated from the date of surgery to last telephonic follow-up.

This study was approved by the Ethical Committee of Shanghai Chest Hospital of Shanghai Jiao Tong University, Shanghai, China. All patients agreed to participate in the study with all relevant data and written informed consent was obtained from all patients before surgery and subsequent clinical studies. Written informed consents were also obtained from all patients or their legal representatives for the use of surgically resected specimens, as appropriate.

\section{Immunohistochemical Analysis}

A panel of immunohistochemical markers, including cytokeratin (1:400 diluted; clone AE1/AE3; Dako), P63 (1:200 diluted; clone DAK-p63; Dako), TDT (ready-to-use; clone EP266; Dako), CD3 (1:200 diluted; clone SP7), CD20 (1:200 diluted; clone MX003), and CD5 (1:200 diluted; clone 4C7; DAKO), and CD117 (readyto-use; Polyclonal; Dako) were used for routine and differential diagnosis in the tissue sections of formalin-fixed paraffin- embedded thymoma specimens. $4-5 \mu \mathrm{m}$ representative tissue sections were used and immunohistochemical analysis was performed using the auto-stainer GI100 (DAKO OMNIS; Agilent technologies) and automated stainer (Ventana Benchmark XT; Roche Ventana) following the manufacturer's instructions. All the samples were stained with $H \& E$ in the first and last slides. Immunostained sections were counterstained with hematoxylin. Appropriate positive and negative controls were concurrently run.

\section{Unified RNA and DNA NGS Assay by PANO-Sequencing Analysis Program}

Selected cases were subjected to the parallel amplification numerically optimized (PANO) sequencing assay (10), using a modified on-shelf product (panel \#022T, HeliTec Biotechnologies, ShenZhen, China) with designed MAML2 primers spiked in the multiplexed primer pools. This panel can identify all functional fusion events in multiple common genes and genetic variations in all the National Comprehensive Cancer Network (NCCN)-specified biomarkers. To perform this assay, total nucleic acids were first extracted from formalin-fixed paraffin-embedded (FFPE) tissue samples using a PANO-Pure FFPE TNA extraction kit (HeliTec Biotechnologies, ShenZhen, China), and $50 \mathrm{ng}$ of input was used for library construction. This single-tube library construction protocol used DNA for the detection of single nucleotide variants (SNV) as well as insertions and detections (InDels) mutations, RNA for fusion detection, and tiled intronic primers for DNA-based fusion detection when transcripts were unavailable. The reactions were performed in a single tube from extraction to sequencing as a unified library, without experimentally separating DNA or RNA. Sequencing were performed. Raw sequencing data were analyzed using a proprietary PANO-Call ver. 18.12 bioinformatics pipeline for both mutation and fusion calls.

\section{Fluorescence In Situ Hybridization (FISH) Analysis for MAML2 Translocation}

Fusion FISH assays were performed on nine metaplastic thymoma cases including six fusion gene-positive cases and three fusion gene-negative cases. $4 \mu \mathrm{m}$ thick representative tissue sections slides of FFPE tissue sections were used. Epithelioid and spindle cell components were laser-microdissected respectively for analysis using a commercial MAML2 Break Apart Rearrangement Kit. All slides were deparaffinized, pretreated, hybridized with denatured probes, and incubated overnight. The slides were then washed and stained with 4',6diamidino-2-phenylindole (DAPI). The results were visualized using an automatic fluorescence microscope (Zeiss AXI0, Imager.Z2, Germany). The SPEC MAML2 Dual Color Break Apart Probe is a mixture of two directly labeled probes hybridizing to the 11q21 band. The green fluorochrome directlabeled probe hybridizes distal to the MAML2 gene, and the orange fluorochrome direct-labeled probe hybridizes proximal to that gene. At least 200 cells were counted for each section. By calculating the number of positive cells greater than three areas 
and taking the average value, those cases with more than $10 \%$ of tumor cells showing positivity were interpreted as harboring $M A M L 2$ gene rearrangement.

\section{RESULTS}

\section{Clinical and Pathologic Findings}

The cohort of 17 metaplastic thymoma patients comprised of nine men and eight women, aged 29-71 years. The tumor size ranged from $1.5 \mathrm{~cm}$ to $13.5 \mathrm{~cm}$. Clinical information and prognosis data are presented in Table 1. All patients underwent surgical treatment and did not receive postoperative adjuvant therapy. The follow-up duration was eight months to ten years, and no case of tumor recurrence was found until the final follow-up. Clinical information on micronodular thymoma with lymphoid stroma and type A thymoma is listed in Supplementary Table 1. Morphologically, most metaplastic thymomas displayed classical histopathology which consisted of solid growth epithelial cell components and mild gradually transitioning spindle cell components in varying proportions (Figure 1). Two cases focally or partially showed characteristic micronodular-like growth patterns with abundant lymphoid stroma. However, unlike in MNTLS, the lymphocytic stroma comprised of almost TDT-positive and CD20-negative T lymphocytes with scattered epithelial tumor cells present in the two cases (Figure 2). In case 5, the biphasic differentiation of the tumor was not obvious and the epithelial component accounted for more than $90 \%$ of the evaluated slides. In case 8 , the tumor cells displayed obvious degenerative changes with strange irregular nuclei, intranuclear pseudo-inclusions, but with no proliferate activity. No correlation was observed between morphological differences and fusion gene status. Immunohistochemically, the epithelial islands in all cases were strongly positive for pan-cytokeratin and P63. CD5, CD117 and CD20 expression were negative. TDT- and CD3-positive lymphocytes were only confined to micronodular-like growth areas. The immunohistochemical staining results for tumor cells and lymphoid stroma are listed in Supplementary Table 2.

\section{Unified RNA and DNA NGS Assay}

Sequencing analysis identified YAP1-MAML2 translocation in 13 out of 17 (76.5\%) cases of metaplastic thymomas but not in MNTLs and type A thymomas. Moreover, two metaplastic thymoma cases with micronodular-like growth patterns were also found to harbor this rearrangement. There was no significant correlation between translocation and the proportion of cellular components or growth patterns in metaplastic thymoma. No other fusion events were detected in any of the cases. Intrachromosomal inversion by RNA sequencing identified five products in metaplastic thymoma: 5 YAP1_exon 1 fused to 3 MAML2_exon 2, 5 YAP1_exon 1 fused to 3 MAML2_exon 3, 5 YAP1_exon 4 fused to 3 MAML2_exon 2, 5 $Y A P 1$ exon 5 fused to 3 MAML2_exon 2 and 5 YAP1_exon 6 fused to 3 MAML2_exon 2. Six nonsense mutations were identified in metaplastic thymoma cases. Four mutations were found in cases with MAML2 fusions: ALK Q1188X in case 1, CDK4 R5X in case 3, PTEN Q110X in case 5, and CDK4 W179X in case 6. The other two nonsense mutations, HRAS Q95X and BRAF W476X, were detected in one fusion-negative patient. These nonsense mutations result in the production of a shortened protein, which may be nonfunctional. Breakpoint locations determined by DNA-based sequencing are listed in Table 2.

\section{Validation of MAML2 Fusions in Metaplastic Thymoma by FISH Verifying}

Tumors of nine patients' harboring fusion conditions including six sequencing fusion-positive cases and three fusion-negative cases were detected by FISH method in microdissection slides. The results revealed that both epithelioid and spindle cell components harboring YAP1-MAML2 fusions in positive cases, including two tumors with micronodular-like growth pattern (Figures 1 and 2).

TABLE 1 | Clinical information of 17 cases of metaplastic thymoma in this study.

\begin{tabular}{|c|c|c|c|c|c|c|c|}
\hline Case no. & Age & Sex & Maximum diameter $(\mathbf{c m})$ & Smoking status & Masaoka-Koga staging & Treatment & Follow-up \\
\hline 1 & 68 & M & 7.1 & 20 packs/years & I & Surgical resection & Alive at 10 years \\
\hline 2 & 35 & $M$ & 5.2 & non & Ila & Surgical resection & Alive at 7 years \\
\hline 3 & 65 & M & 13 & 200 packs/year & I & Surgical resection & Alive at 4.5 years \\
\hline 4 & 57 & $\mathrm{~F}$ & 2.5 & non & Ila & Surgical resection & Alive at 3.5 years \\
\hline 5 & 29 & $\mathrm{~F}$ & 8 & non & 1 & Surgical resection & Alive at 3.5 years \\
\hline 6 & 69 & $\mathrm{~F}$ & 5 & non & Ila & Surgical resection & Alive at 3 years \\
\hline 7 & 57 & $\mathrm{~F}$ & 4 & non & Ila & Surgical resection & Alive at 2.5 years \\
\hline 8 & 60 & $M$ & 3.6 & 50 packs/year & I & Surgical resection & Alive at 2.5 years \\
\hline 9 & 36 & $\mathrm{~F}$ & 4 & non & I & Surgical resection & Alive at 2 years \\
\hline 10 & 31 & $M$ & 7.8 & non & Ilb & Surgical resection & Alive at 2 years \\
\hline 11 & 45 & $M$ & 2.2 & non & Ila & Surgical resection & Alive at 1.5 years \\
\hline 12 & 52 & $M$ & 1.5 & non & $\mathrm{Ila}$ & Surgical resection & Alive at 1.5 years \\
\hline 13 & 71 & $\mathrm{~F}$ & 5.2 & non & Ila & Surgical resection & Alive at 1.5 years \\
\hline 14 & 55 & $\mathrm{~F}$ & 7 & non & Ila & Surgical resection & Alive at 1 years \\
\hline 15 & 52 & $M$ & 13.5 & 40 packs/year & I & Surgical resection & Alive at 1 years \\
\hline 16 & 31 & $\mathrm{~F}$ & 3 & non & Ila & Surgical resection & Alive at 1 years \\
\hline 17 & 32 & $M$ & 10 & non & $\mathrm{Ila}$ & Surgical resection & Alive at 8 months \\
\hline
\end{tabular}




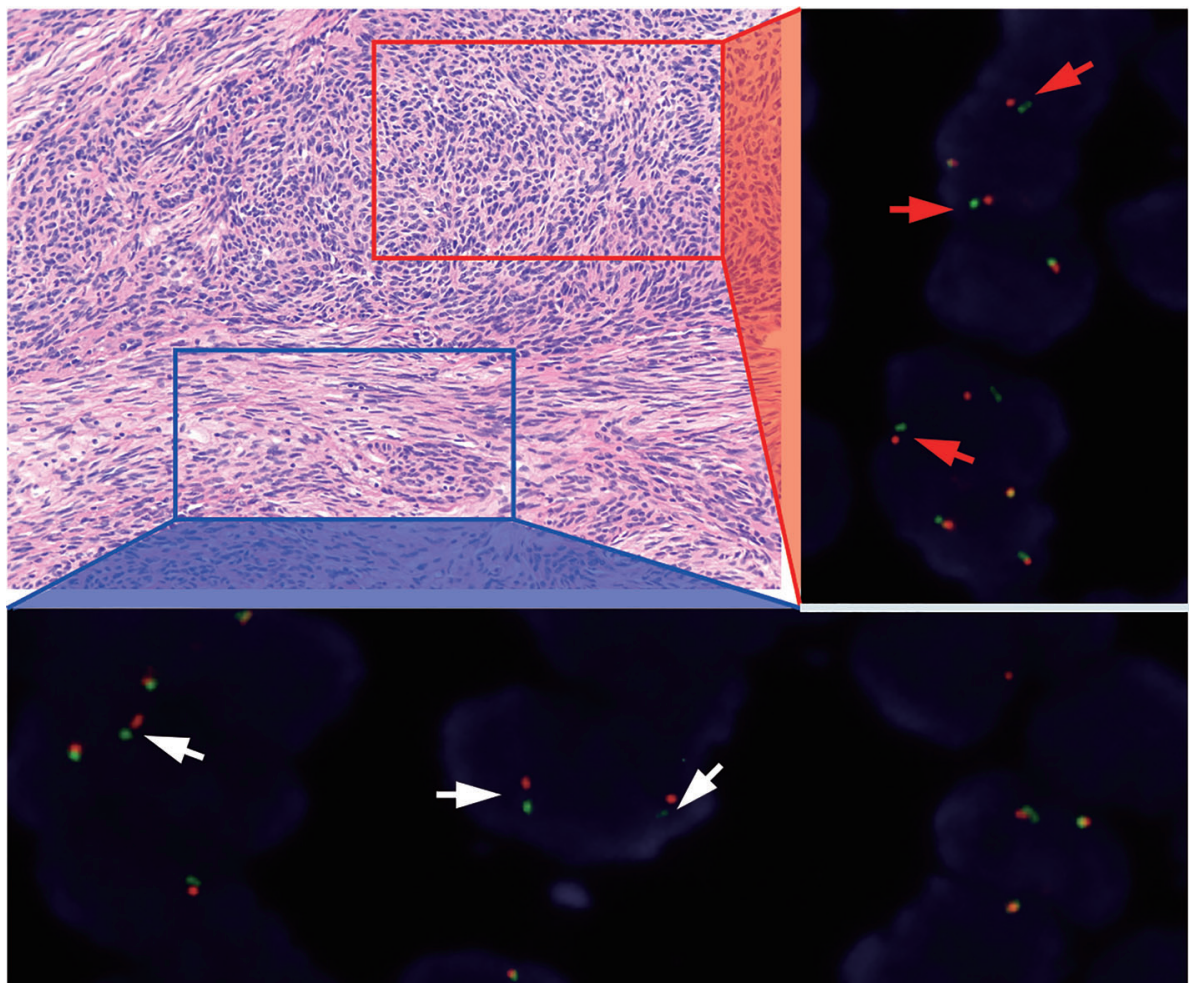

FIGURE 1 | Histological features of metaplastic thymoma. Anastomosing cords or trabeculae of epithelioid cell (red rectangle) and pseudosarcomatous spindle cell components (blue rectangle). H\&E staining at 40X magnification. Both epitheloid and spindle cell components harboring YAP1-MAML2 gene rearrangements are indicated by red and white arrows respectively.
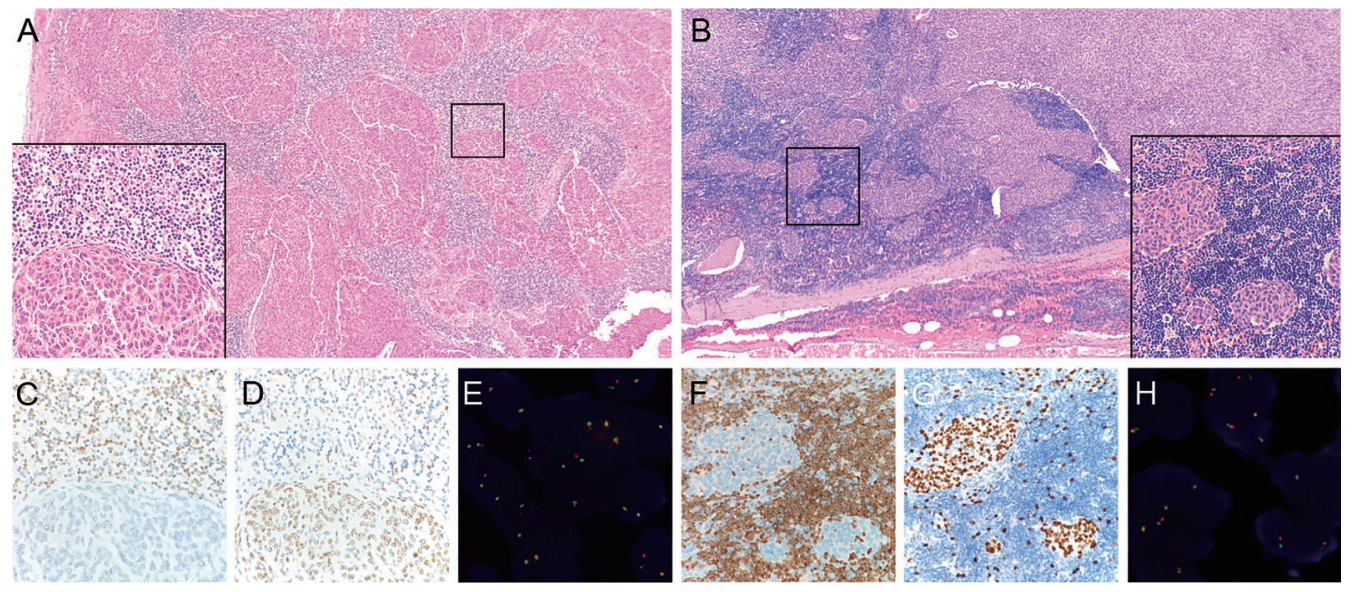

FIGURE 2 | Micronodular-like growth pattern in metaplastic thymoma. (A) This growth pattern accounts for 50\% of the tumor size in case 5, H\&E staining at 4X magnification. The box in the left bottom shows a clear border between epithelioid cell nests and lymphoid stroma, H\&E staining at 40X magnification.

(B) Micronodular-like growth pattern is focally present in case 10, H\&E staining at $4 \mathrm{X}$ magnification. (C) TDT-positive lymphocytes are not present in epithelial cell components of case 5. (D, G) P63 outline the epithelial cells. (F) TDT-positive lymphocytes are scattered in epithelioid cell nests of case 10. (E, H) Micronodular-like growth epithelioid cell nests all display narrow split signals in the assays using MAML2 dual color break apart probe. 
TABLE 2 | Unified RNA and DNA NGS Assay and FISH detection of YAP1-MAML2 fusions in 17 metaplastic thymoma cases.

\begin{tabular}{|c|c|c|c|c|c|c|c|c|}
\hline \multirow[t]{2}{*}{ Case no. } & \multicolumn{3}{|c|}{ Cellular components } & \multicolumn{2}{|c|}{ RNA fusions data } & \multicolumn{2}{|c|}{ DNA sequencing data } & \multirow[t]{2}{*}{ FISH result } \\
\hline & Epithelioid (\%) & Spindle (\%) & micronodular-like (\%) & $5^{\prime}$ partner & $3^{\prime}$ partner & YAP1 breakpoint & MAML2 breakpoint & \\
\hline 1 & 60 & 40 & None & YAP1_exon1 & MAML2_exon2 & 11_101981900 & 11_95826684 & None detected \\
\hline 2 & 50 & 50 & None & YAP1_exon4 & MAML2_exon2 & 11_102076805 & 11_95826684 & None detected \\
\hline 3 & 70 & 30 & None & YAP1_exon4 & MAML2_exon2 & 11_102076805 & 11_95826684 & Positive \\
\hline 4 & 70 & 30 & None & YAP1_exon1 & MAML2_exon2 & 11_101981900 & 11_95826684 & None detected \\
\hline 5 & 40 & 10 & 50 & YAP1_exon4 & MAML2_exon2 & 11_102076805 & 11_95826684 & Positive \\
\hline 6 & 60 & 40 & None & YAP1_exon1 & MAML2_exon2 & 11_101981869 & 11_95826682 & None detected \\
\hline 7 & 60 & 40 & None & YAP1_exon4 & MAML2_exon2 & 11_102076805 & 11_95826684 & None detected \\
\hline 8 & 70 & 30 & None & None & None & None & None & Negative \\
\hline 9 & 80 & 20 & None & YAP1_exon1 & MAML2_exon2 & 11_101981900 & 11_95826684 & None detected \\
\hline 10 & 50 & 40 & 10 & YAP1_exon1 & MAML2_exon2 & 11_101981900 & 11_95826684 & Positive \\
\hline 11 & 90 & 10 & None & None & None & None & None & None detected \\
\hline 12 & 60 & 40 & None & $\begin{array}{l}\text { YAP1_exon4 } \\
\text { YAP1_exon6 }\end{array}$ & $\begin{array}{l}\text { MAML2_exon2 } \\
\text { MAML2_exon2 }\end{array}$ & 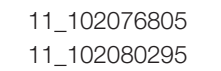 & $\begin{array}{l}\text { 11_95826684 } \\
\text { 11_95826685 }\end{array}$ & Positive \\
\hline 13 & 70 & 30 & None & None & None & None & None & Negative \\
\hline 14 & 60 & 40 & None & YAP1_exon1 & MAML2_exon2 & 11_101981900 & 11_95826684 & None detected \\
\hline 15 & 80 & 20 & None & $\begin{array}{l}\text { YAP1_exon4 } \\
\text { YAP1_exon5 } \\
\text { YAP1_exon6 }\end{array}$ & $\begin{array}{l}\text { MAML2_exon2 } \\
\text { MAML2_exon2 } \\
\text { MAML2_exon2 }\end{array}$ & 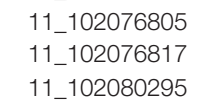 & $\begin{array}{l}\text { 11_95826684 } \\
\text { 11_95826684 } \\
\text { 11_95826685 }\end{array}$ & Positive \\
\hline 16 & 80 & 20 & None & $\begin{array}{l}\text { YAP1_exon1 } \\
\text { YAP1_exon1 }\end{array}$ & $\begin{array}{l}\text { MAML2_exon2 } \\
\text { MAML2_exon3 }\end{array}$ & $\begin{array}{l}11 \_101981900 \\
11 \_101981901\end{array}$ & $\begin{array}{l}\text { 11_95826684 } \\
\text { 11_95724889 }\end{array}$ & Positive \\
\hline 17 & 50 & 50 & None & None & None & None & None & Negative \\
\hline
\end{tabular}

\section{DISCUSSION}

Our molecular findings confirmed a higher frequency of YAP1$M A M L 2$ gene rearrangements in metaplastic thymomas. In addition, six nonsense mutations were identified in these cases, including ALK Q1188X, CDK4 R5X, PTEN Q110X, and CDK4 W179X in four fusion gene-positive cases, and HRAS Q95X along with $B R A F \mathrm{~W} 476 \mathrm{X}$ in one fusion gene-negative case. The MAML2 gene rearrangements were also studied in micronodular thymoma with lymphoid stroma and conventional type A thymoma, but no rearrangements were found in these types. Furthermore, we identified a micronodular-like growth pattern in two cases of metaplastic thymoma.

Thymic epithelial tumors are traditionally classified into four groups: spindle cell thymoma, predominantly lymphocytic thymoma, mixed lymphocytic and epithelial thymoma, and predominantly epithelial thymoma, according to the lymphocyte-to-epithelial cell ratio and the shape of epithelial cells (11). Taking into account the prognosis and molecular alterations, the $2004^{\text {th }}$ and $2015^{\text {th }}$ WHO classification of tumors of the thymus modified and revised the nomenclature $(4,12)$. Occasionally, in addition to spindle cell morphology, type A thymoma demonstrates various histological patterns including rosettes with or without central lumens, papillary projections in cystic spaces, or meningioma-like wholes, and may mimic a biphasic growth pattern that is often devoid of immature T cells. In our cohort, we also identified cases of metaplastic thymoma with abundant lymphoid stroma. Two patients had abundant lymphoid stroma mimicking the growth pattern of micronodular thymoma, and one patient had symptoms of slight blepharoptosis. Unlike MNTLS in which most of the lymphocytes, were mature B lymphocytes, the lymphoid cells of the stroma in these two metaplastic thymoma cases were
TDT-positive T lymphocytes, and these lymphocytes would or would not be present in the epithelial nests. However, scattered tumor epithelial cells could always be found in the lymphoid stroma, reminiscent of type B1 thymoma. Miki et al. have suggested a relationship between metaplastic thymoma and type $A B$ thymoma (13). Therefore, it is necessary to distinguish metaplastic thymoma from type $A$ and $B$ in clinical practice, as it is quite challenging in individual cases by only morphological evaluation.

The epithelial-mesenchymal transition seems to be involved in the transitional differentiation of epithelioid and spindle cell components. Moreover, the histogenesis and relationship between metaplastic thymoma and other thymic epithelial neoplasms remains unknown (14). Sarcomatoid transformation was not found in metaplastic thymoma cases that were included in our study as per the follow-up data available, and this phenomenon has been reported in very few studies $(15,16)$.

There may be morphological or even genetic alterations that overlap between metaplastic thymoma, type A/AB and MNTLS, but chromosomal abnormalities by comparative genomic hybridization and previous molecular studies have shown no evidence to support these conjectures (17). Marina et al. have reported a recurrent genetic signature of YAP1-MAML2 fusion in metaplastic thymoma for the first time and described two distinct YAP1-MAML2 transcription products (9). Our results confirm a high frequency of YAP1-MAML2 fusions in metaplastic thymoma cases, suggesting that it may be a common pathogenetic mechanism in the development of metaplastic thymomas. Although there was a certain degree of morphological overlap among metaplastic thymoma, MNTLS or even type $\mathrm{A} / \mathrm{AB}$ thymoma, it basically did not affect the accurate pathological typing. In addition, sequencing results have also suggested that the histogenesis mechanism of metaplastic 
thymoma may be different from MNTLS and type A/AB thymoma. In a recent study, Lucas et al. have described a recurrent gene rearrangement of KMT2A-MAML2 in type B2 and B3 thymomas (18). Fusion with different gene partners suggests that MAML2 gene rearrangement may be a potential biomarker for the morphological classification of thymomas.

Recurrent YAP1-MAML2 fusions have been reported in poroma and porocarcinomas, pediatric NF2-wildtype meningioma, composite and retiform hemangioendothelioma, glioblastoma, nasopharyngeal carcinoma and ovarian cancer cell lines (19-24). However, its function is poorly understood. YAP1 and $M A M L 2$ are on the opposite strands of adjacent genetic loci on the $\mathrm{p}$ arm chromosome 11, and the co-expression of YAP1$M A M L 2$ fusion transcripts is thought to be the consequence of intrachromosomal inversions. Gabriele et al. have validated the fusion and found that YAP1-MAML2 fusion is associated with increased YAP1 signaling and further driving the Hippo signaling cascade in YAP1-MAML2 tumors (25). Previous studies on multiple solid tumors suggest that YAP1 aberrant activation is related to poor prognosis, chemoresistance, and resistance to cell death $(26,27)$. However, the biologic behavior of metaplastic thymoma appears indolent, and the function of YAP1-MAML2 fusion in metaplastic thymoma needs to be further explored.

In conclusion, the present study further confirms and emphasizes the fundamental genetic alterations of YAP1$M A M L 2$ gene rearrangements in metaplastic thymomas. Moreover, we identified two cases of metaplastic thymoma with a micronodular growth pattern. Our findings also indicate that the YAP1-MAML2 gene rearrangements may be a useful diagnostic biomarker for distinguishing metaplastic thymoma from type A thymoma and micronodular thymoma with lymphoid stroma.

\section{DATA AVAILABILITY STATEMENT}

The datasets presented in this study can be found in online repositories. The names of the repository/repositories and accession number(s) can be found in the article/Supplementary Material.

\section{REFERENCES}

1. Liu B, Rao Q, Zhu Y, Yu B, Zhu HY, Zhou XJ. Metaplastic Thymoma of the Mediastinum: A Clinicopathologic, Immunohistochemical, and Genetic Analysis. Am J Clin Pathol (2012) 137(2):261-9. doi: 10.1309/ AJCP0T1JFYLMPHMI

2. Yoneda S, Marx A, Müller-Hermelink HK. Low-Grade Metaplastic Carcinomas of the Thymus: Biphasic Thymic Epithelial Tumors With Mesenchymal Metaplasia-an Update. Pathol Res Pract (1999) 195:555-63. doi: 10.1016/S0344-0338(99)80005-3

3. Travis WD, Brambilla E, Müller-Hermelink HK. WHO Classification of Tumours: Pathology and Genetics of Tumours of the Lung, Pleura, Thymus and Heart. Lyon, France: IARC Press (2004) p. 146-71.

4. Travis WD, Brambilla E, Burke AP, Marx Z, Nicholson AG. WHO Classification of Tumours of the Lung, Pleura, Thymus and Heart. 4th edn. Lyon France: IARC (2015) p. 187-209.

5. Jin M, Liu B, Wang L, Xu JY. Clinicopathologic Study of Metaplastic Thymoma. Zhonghua Bing Li Xue Za Zhi (2006) 35:285-8. doi: 10.3760/ j.issn:0529-5807.2006.05.008

\section{ETHICS STATEMENT}

The studies involving human participants were reviewed and approved by Ethical Committee of Shanghai Chest Hospital of Shanghai Jiao Tong University. The patients/participants provided their written informed consent to participate in this study.

\section{AUTHOR CONTRIBUTIONS}

YH designed the study. RZ and LG performed next-generation sequencing and analyzed the data of FISH. JZ, JS, and $\mathrm{YH}$ performed morphological evaluation and LG completed immunohistochemical staining. RZ and CX analyzed and interpreted the sequencing data. JZ wrote the main manuscript. $\mathrm{YH}$ and $\mathrm{RZ}$ participated in the writing part of the discussion. JZ and RZ contributed equally to this work. All authors contributed to the article and approved the submitted version.

\section{FUNDING}

The study was funded by Nurture Projects for Basic Research of Shanghai Chest Hospital (Grant number: 2020YNJCM13).

\section{SUPPLEMENTARY MATERIAL}

The Supplementary Material for this article can be found online at: https://www.frontiersin.org/articles/10.3389/fonc.2021.692283/ full\#supplementary-material

Supplementary Table 1 | Clinical information of micronodular thymoma with lymphoid stroma (MNTLS) and type A thymoma cases used in this study.

Supplementary Table 2 | The immunohistochemical staining results for tumor cells and lymphocytes components in metaplastic thymoma.
6. Kang G, Yoon N, Han J, Kim YE, Kim TS, Kim K. Metaplastic Thymoma: Report of 4 Cases. Korean J Pathol (2012) 46:92-5. doi: 10.4132/KoreanJPathol.2012.46.1.92

7. Poorabdollah M. Metaplastic Thymoma: Report of an Unusual Thymic Epithelial Neoplasm Arising in the Wall of a Thymic Cyst. Int J Surg Pathol (2009) 17:51-4. doi: 10.1177/1066896908315754

8. Tajima S, Yanagiya M, Sato M, Nakajima J, Fukayama M. Metaplastic Thymoma With Myasthenia Gravis Presumably Caused by an Accumulation of Intratumoral Immature T Cells: A Case Report. Int J Clin Exp Pathol (2015) 8:15375-80.

9. Vivero M, Davineni P, Nardi V, Chan JKC, Sholl LM. Metaplastic Thymoma: A Distinctive Thymic Neoplasm Characterized by YAP1-MAML2 Gene Fusions. Modern Pathol (2020) 33:560-5. doi: 10.1038/s41379-019-0382-x

10. Song Z, Xu C, He Y, Li F, Wang W, Zhu Y, et al. Simultaneous Detection of Gene Fusions and Base Mutations in Cancer Tissue Biopsies by Sequencing Dual Nucleic Acid Templates in Unified Reaction. Clin Chem (2020) 66:17887. doi: $10.1373 /$ clinchem.2019.308833

11. Lattes R. Thymoma and Other Tumours of the Thymus: An Analysis of 107 Cases. Cancer (1962) 15:1224-60. doi: 10.1002/1097-0142(196211/12) 15:6<1224::AID-CNCR2820150621>3.0.CO;2-V 
12. Ströbel P, Marx A, Zettl A, Müller-Hermelink HK. Thymoma and Thymic Carcinoma: An Update of the WHO Classification 2004. Surg Today (2005) 35:805-11. doi: 10.1007/s00595-005-3047-y

13. Miki YHK, Yoshino T, Miyatani K, Takahashi K. Type AB Thymoma Is Not a Mixed Tumor of Type A and Type B Thymomas, But a Distinct Type of Thymoma. Virchows Arch (2014) 464:725-34. doi: 10.1007/s00428-0141587-5

14. Mohamet L, Hawkins K, Ward CM. Loss of Function of E-Cadherin in Embryonic Stem Cells and the Relevance to Models of Tumorigenesis. J Oncol (2011) 2011:1687-706. doi: 10.1155/2011/352616

15. Moritani S, Ichihara S, Mukai K, Seki Y, Inoue S, Yasuda A, et al. Sarcomatoid Carcinoma of the Thymus Arising in Metaplastic Thymoma. Histopathology (2008) 52:409-11. doi: 10.1111/j.1365-2559.2007.02960.x

16. Lu HS, Gan MF, Zhou T, Wang SZ. Sarcomatoid Thymic Carcinoma Arising in Metaplastic Thymoma: A Case Report. Int J Surg Pathol (2011) 19:677-80. doi: 10.1177/1066896909355458

17. Radovich M, Pickering CR, Felau I, Ha G, Zhang H, Jo H, et al. The Integrated Genomic Landscape of Thymic Epithelial Tumors. Cancer Cell (2018) 33:24458. doi: 10.1016/j.ccell.2018.01.003

18. Massoth LR, Hung YP, Dias-Santagata D, Onozato M, Shah N, Severson E, et al. Pan-Cancer Landscape Analysis Reveals Recurrent KMT2A - MAML2 Gene Fusion in Aggressive Histologic Subtypes of Thymoma. JCO Precis Oncol (2020) 4:109-15. doi: 10.1200/PO.19.00288

19. Sekine S, Kiyono T, Ryo E, Ogawa R, Wakai S, Ichikawa H, et al. Recurrent YAP1-MAML2 and YAP1-NUTM1 Fusions in Poroma and Porocarcinoma. J Clin Investig (2019) 130:3827-32. doi: 10.1172/JCI126185

20. Agaimy A, Stoehr R, Tögel L, Hartmann A, Cramer T. YAP1-MAML2Rearranged Poroid Squamous Cell Carcinoma (Squamoid Porocarcinoma) Presenting as a Primary Parotid Gland Tumor. Head Neck Pathol (2021) 15:361-7. doi: 10.1007/s12105-020-01181-9

21. Sievers P, Chiang J, Schrimpf D, Stichel D, Paramasivam N, Sill M, et al. YAP1-Fusions in Pediatric NF2-Wildtype Meningioma. Acta Neuropathol (2020) 139:215-8. doi: 10.1007/s00401-019-02095-9
22. Antonescu CR, Dickson BC, Sung YS, Zhang L, Suurmeijer AJH, Stenzinger A, et al. Recurrent YAP1 and MAML2 Gene Rearrangements in Retiform and Composite Hemangioendothelioma. Am J Surg Pathol (2020) 44:1677-84. doi: 10.1097/PAS.0000000000001575

23. Valouev A, Weng Z, Sweeney RT, Varma S, Le QT, Kong C, et al. Discovery of Recurrent Structural Variants in Nasopharyngeal Carcinoma. Genome Res (2014) 24:300-9. doi: 10.1101/gr.156224.113

24. Papp E, Hallberg D, Konecny GE, Bruhm DC, Adleff V, Noë M, et al. Integrated Genomic, Epigenomic, and Expression Analyses of Ovarian Cancer Cell Lines. Cell Rep (2018) 25:2617-33. doi: 10.1016/j.celrep.2018.10.096

25. Picco G, Chen ED, Alonso LG, Behan FM, Gonçalves E, Bignell G, et al. Functional Linkage of Gene Fusions to Cancer Cell Fitness Assessed by Pharmacological and CRISPR-Cas9 Screening. Nat Commun (2019) 10:2198. doi: 10.1038/s41467-019-09940-1

26. Lee KW, Lee SS, Kim SB, Sohn BH, Lee HS, Jang HJ, et al. Significant Association of Oncogene YAP1 With Poor Prognosis and Cetuximab Resistance in Colorectal Cancer Patients. Clin Cancer Res (2015) 21:357-64. doi: 10.1158/1078-0432.CCR-14-1374

27. Panciera T, Azzolin L, Cordenonsi M, Piccolo S. Mechanobiology of YAP and TAZ in Physiology and Disease. Nat Rev Mol Cell Biol (2017) 18:758-70. doi: $10.1038 /$ nrm.2017.87

Conflict of Interest: The authors declare that the research was conducted in the absence of any commercial or financial relationships that could be construed as a potential conflict of interest.

Copyright (c) 2021 Zhao, Zhao, Xiang, Shao, Guo and Han. This is an open-access article distributed under the terms of the Creative Commons Attribution License (CC BY). The use, distribution or reproduction in other forums is permitted, provided the original author(s) and the copyright owner(s) are credited and that the original publication in this journal is cited, in accordance with accepted academic practice. No use, distribution or reproduction is permitted which does not comply with these terms. 